\title{
Application of extended self-similarity in turbulence
}

\author{
Siegfried Grossmann, Detlef Lohse, and Achim Reeh \\ Fachbereich Physik der Universität Marburg, Renthof 6, D-35032 Marburg, Germany
}

(Received 10 April 1997; revised manuscript received 14 July 1997)

\begin{abstract}
From Navier-Stokes turbulence numerical simulations we show that for the extended self-similarity (ESS) method it is essential to take the third order structure function taken with the modulus and called $D_{3}^{*}(r)$, rather than the standard third order structure function $D_{3}(r)$ itself. If this is done, we find ESS towards scales larger than order $\sim 10 \eta$, where $\eta$ is the Kolmogorov scale. If $D_{3}(r)$ is used, there is no ESS. We also analyze ESS within the Batchelor parametrization of the second and third order longitudinal structure function and focus on the scaling of the transversal structure function. The Re-asymptotic inertial range scaling develops only beyond a Taylor-Reynolds number $\operatorname{Re}_{\lambda} \geq 500$. [S1063-651X(97)05311-7]

PACS number(s): 47.27.Gs, 47.27.Eq, 47.27.Jv
\end{abstract}

\section{INTRODUCTION}

Extended self-similarity (ESS, [1-3]) has been most useful in determining scaling exponents in experimental and numerical turbulent flow. In ESS, the $p$ th order longitudinal velocity structure function

$$
D_{p}^{L}(r)=\left\langle\left\{[\boldsymbol{u}(\boldsymbol{x}+\boldsymbol{r})-\boldsymbol{u}(\boldsymbol{x})] \cdot \boldsymbol{e}_{r}^{L}\right\}^{p}\right\rangle
$$

is plotted against the third order structure function. Here, $\boldsymbol{e}_{r}^{L}$ is the unit vector in $\boldsymbol{r}$ direction; the unit vector $\boldsymbol{e}_{r}^{T}$ used below is perpendicular to $\boldsymbol{e}_{r}^{L}$. The original motivation for picking the third order structure function was the Howard-von Kármán-Kolmogorov structure equation [4-6]

$$
D_{3}^{L}(r)=-\frac{4}{5} \epsilon r+6 \nu \frac{d}{d r} D_{2}^{L}(r),
$$

saying that in the inertial subrange (ISR) $D_{3}^{L}(r)$ scales as $D_{3}^{L} \propto r$ and therefore $D_{p}^{L} \propto r_{p}^{L} \propto\left[D_{3}^{L}(r)\right]_{p}^{L}$ has the same scaling exponent as a function of $r$ or of $D_{3}^{L}$. However, because of the poor statistical convergence, rather than $D_{3}^{L}(r)$, the third order structure function $D_{3}^{* L}(r)$, calculated with the modulus of the velocity difference, is taken and it is argued $[1,3]$ that $D_{3}^{* L}(r)$ would also scale linearly with $r$ in the ISR [7]. The resulting exponents, which in general have to be distinguished from the $\zeta_{p}^{L}$, s [8], are denoted as $\xi_{p}^{L}$, defined by $D_{p}^{L} \propto\left(D_{3}^{* L}\right)^{\xi_{p}^{L}}$, and they are found to be remarkably universal, i.e., independent of flow geometry and Reynolds number $[9,10]$.

Note that the degree of intermittency could be quantified by plotting $D_{p}^{L}(r)$ vs any structure function $D_{q}^{L}(r)$ [odd order moments taken with the modulus, $\left.D_{q}^{* L}(r)\right]$.

In this paper we would like to demonstrate that-beyond the mere practical reason of better statistics - it seems really essential for physical reasons to take $D_{3}^{* L}(r)$ rather than $D_{3}^{L}(r)$ to have ESS. We do so by examining ESS both for a full numerical simulation [11] and for Batchelor's parametrization of the structure function [12]. This parametrization will give us the opportunity to study finite Reynolds number
Re effects. We will furthermore discuss the difference in scaling of the longitudinal as compared to the transversal structure functions.

We numerically solve the three dimensional (3D) incompressible Navier-Stokes equation on an $N^{3}$ grid with periodic boundary conditions. A pseudospectral code is used, the flow is forced on the largest length scales. For $N=96$ we achieve a Taylor-Reynolds number $\operatorname{Re}_{\lambda}=110$; scales down to $3 \eta$ are resolved; $\eta=\nu^{3 / 4} / \epsilon^{1 / 4}$ is the Kolmogorov length, $\nu$ the kinematic viscosity, $\epsilon$ the energy dissipation rate. Time integrations up to 150 large eddy turnovers are performed; the flow is locally isotropic to a high degree. More details on the numerical flow are given in Ref. [11].

\section{BATCHELOR'S PARAMETRIZATION}

The longitudinal second order structure function $D_{2}^{L}(r)$ is shown in Fig. 1. As in the whole paper, lengths are given in multiples of $\eta$ and velocities in multiples of $(\epsilon \eta)^{1 / 3}$. An ISR scaling range is hardly developed because $\operatorname{Re}_{\lambda}=110$ is still small. The data are very well fitted by a parametrization of Batchelor's type [12-16] with an additional large scale cutoff $L[15,17]$,

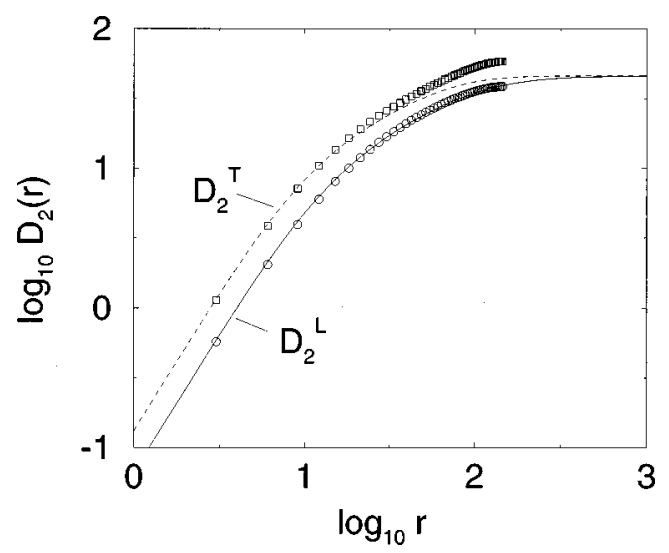

FIG. 1. Batchelor parametrization (3) (solid line) of the $N=96$ data for the longitudinal second order structure function $D_{2}^{L}(r)$ (numerical data: circles). We chose $a=12.4 \eta$ and $L=108 \eta$. Then we used Eq. (6) to calculate $D_{2}^{T}(r)$ (dashed line) which poorly compares with the numerical data (squares) for $r$ beyond the VSR. 


$$
D_{2}^{L}(r)=\frac{\epsilon}{15 \nu} \frac{r^{2}}{\left[1+(r / a)^{2}\right]^{1-\zeta / 2}} \frac{1}{\left[1+(r / L)^{2}\right]^{\zeta / 2}} .
$$

Here, $\zeta=\zeta_{2}$ is the asymptotic ISR scaling exponent which from our [11] and others' $[1-3,18,6]$ ESS analysis we take to be $\zeta=0.70$. The only other free parameters are the viscous subrange-inertial subrange (VSR-ISR) crossover scale $a$ and, of course, the large scale cutoff $L$. From a fit of Eq. (3) to the present numerical data we find $a=12.4 \eta$ and $L=108 \eta$.

Note that we do not want to imply that all flow fields show a large scale saturation of type (3). However, those data (both numerical and experimental) we analyzed (see also $[15,17]$ ) were well described by Eq. (3). As Eq. (3) guarantees an analytic behavior of both the correction term to $r^{2}$ on the small scale side and the correction term to $r=$ const on the large scale side we think that this is not accidental.

With the outer length scale $L$ we can define a Reynolds number $\operatorname{Re}=u_{1, \mathrm{rms}} L / \nu$. For very large $\operatorname{Re}$ the structure function (3) develops an ISR scaling law

$$
D_{2}^{L}(r)=b^{L}(\epsilon r)^{2 / 3}\left(\frac{r}{L}\right)^{\delta \zeta_{2}} .
$$

Here, $\delta \zeta_{2}=\zeta_{2}-2 / 3$ is the scaling correction to classical scaling and $b^{L}$ is often called the Kolmogorov constant. From

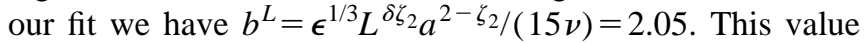
agrees well with the data $b^{L}=1.6-2.5$ known from the literature $[4,13,5,6,19]$; Sreenivasan [19] gives $b^{L}=2.0 \pm 0.4$. Note that the full structure function $D_{2}(r)$ $=3 D_{2}^{L}(r)+r \partial D_{2}^{L} / d r$ (for isotropic flow) asymptotically scales with a law of type (4), too; the prefactor is $b \approx 11 b^{L} / 3=6-9$. Alternatively, also $D_{2}(r)$ can be fitted by a Batchelor parametrization $[13,15]$ with similar quality [15].

Our motivation to employ Batchelor's parametrization (3) is to be able to scale up the second order structure function $D_{2}^{L}(r)$ to much larger $\operatorname{Re}$ (assuming that $b^{L}$ and $\zeta$ are fixed at $b^{L}=2.0$ and $\zeta=0.70$ ) and thereby get consistent data for the transversal second order structure function

$$
D_{p}^{T}(r)=\left\langle\left\{[\boldsymbol{u}(\boldsymbol{x}+\boldsymbol{r})-\boldsymbol{u}(\boldsymbol{x})] \cdot \boldsymbol{e}_{r}^{T}\right\}^{p}\right\rangle,
$$

$p=2$, and for the third order longitudinal structure function $D_{3}^{L}(r)$, which for isotropic, homogeneous, incompressible turbulence both follow from $D_{2}^{L}(r)$, namely, through the relation

$$
D_{2}^{T}(r)=D_{2}^{L}(r)+\frac{r}{2} \frac{d}{d r} D_{2}^{L}(r)
$$

and through Eq. (2), respectively.

To scale up $D_{2}^{L}(r)$ in Eq. (3), we must know how the parameters $a$ and $L$ depend on the Reynolds number. If one accepts Sreenivasan's observations that neither the (asymptotic) dimensionless energy dissipation rate [20-22] $c_{\epsilon}=\epsilon L / u_{1, \mathrm{rms}}^{3}$, nor the Kolmogorov constant $b^{L}$ [19] (but note also Ref. [23]) depend on the Reynolds number, one gets a weak dependence of the VSR-ISR crossover on the Reynolds number [22],

$$
\frac{a}{\eta}=\left(15 b^{L}\right)^{3 /(4-3 \delta \zeta)}\left(\frac{\eta}{L}\right)^{3 \delta \zeta /(4-3 \delta \zeta)}
$$

Once $b^{L}, \zeta$, and $\operatorname{Re}$ are fixed, $L$ and $\operatorname{Re}_{\lambda}$ can easily be obtained from the above equations [21,22]. With $D_{2}^{L}(\infty)=2 u_{1, \mathrm{rms}}^{2}$ we get

$$
\begin{gathered}
\frac{L}{\eta}=\left(\frac{2}{b^{L}}\right)^{3 / 8} \operatorname{Re}^{3 / 4}, \\
\operatorname{Re}_{\lambda}=\frac{\sqrt{15} u_{1, \mathrm{rms}}^{2}}{\sqrt{\nu \epsilon}}=\sqrt{15}\left(\frac{b^{L}}{2}\right)^{3 / 4} \sqrt{\operatorname{Re} .}
\end{gathered}
$$

Vice versa, once $a$ and $L$ are known, we obtain $\operatorname{Re}$ and $\operatorname{Re}_{\lambda}$. For the above values the result is $\operatorname{Re}=520$ and $\operatorname{Re}_{\lambda}=90$ for our numerical flow, in reasonable agreement with the direct numerical result $\operatorname{Re}_{\lambda}=110$. As we will see, the reason for the (modest) underestimation is that in the numerical flow there are correlations left at the largest length scales.

\section{III. $D_{2}^{T}$ AND $D_{3}^{L}$ RESULTING FROM BATCHELOR'S PARAMETRIZATION}

For $D_{2}^{T}(r)$ we find poor agreement between the curve evaluated from Eq. (6) and the numerically obtained values, see Fig. 1. The reason is that at $r \sim L$ there still is considerable correlation $\langle\boldsymbol{u}(\boldsymbol{x}+\boldsymbol{r}) \boldsymbol{u}(\boldsymbol{x})\rangle \neq 0$. More precisely, at the maximal meaningful distance $r_{\max }$ when employing periodic boundary conditions, namely, when $r$ equals half of the periodicity length (here, $r_{\max } \approx 146 \eta$ ), we find $\left\langle u_{j}\left(\boldsymbol{x}+\boldsymbol{e}_{r}^{L} r_{\max }\right) \quad u_{j}(\boldsymbol{x})\right\rangle /\left\langle u_{j}^{2}(\boldsymbol{x})\right\rangle \approx 0.25 \quad$ and $\left\langle u_{j}\left(\boldsymbol{x}+\boldsymbol{e}_{r}^{T} r_{\text {max }}\right) u_{j}(\boldsymbol{x})\right\rangle /\left\langle u_{j}^{2}(\boldsymbol{x})\right\rangle \approx-0.15$ for $j=1$, 2, or 3 . Therefore $D_{2}^{L}\left(r_{\max }\right)$ is smaller and $D_{2}^{T}\left(r_{\max }\right)$ is larger than $2\left\langle u_{j}^{2}\right\rangle$, which is the value the structure functions would take for perfect decorrelation between $\boldsymbol{x}$ and $\boldsymbol{x}+\boldsymbol{r}_{\max }$. Geometrically, the above correlations mean that there is an eddy with diameter $r \sim r_{\max } \sim \pi L$ in the numerical flow. The possibility of such large eddies is a consequence of the periodic boundary conditions (in contrast to boundary conditions which put the velocity to zero at the edge of the flow volume) and will also survive for larger $\mathrm{Re}_{\lambda}$. Such a large scale eddy implies that the flow is not isotropic and homogeneous at the large scales and therefore it should be no surprise that Eq. (6), whose derivation requires isotropy and homogeneity, does not lead to good agreement with the data at large scales. Note that the same problem occurs in experimental flow, see, e.g., Fig. 1(a) of Ref. [24]. At the largest measured distance $r \approx 650 \eta$ (for $\operatorname{Re}_{\lambda}=300$ ) it clearly is $D_{2}^{T}(r)>D_{2}^{L}(r)$, revealing a large scale eddy.

Also for $D_{3}^{L}(r)$ the agreement between the direct numerical values and those from the analytic equation (2), which assumes isotropy and homogeneity, is poor, see Fig. 2. The numerical structure function $D_{3}^{L}(r)$ bends down for large $r$ as the velocity differences at large scales have Gaussian-like statistics and consequently odd order moments almost vanish. This feature which is not described by Eq. (2) is due to the boundary effects; more precisely, because there are no larger eddies than on the scale $r_{\max }$ which could provide correlations. In principle, this deficiency can be cured by 


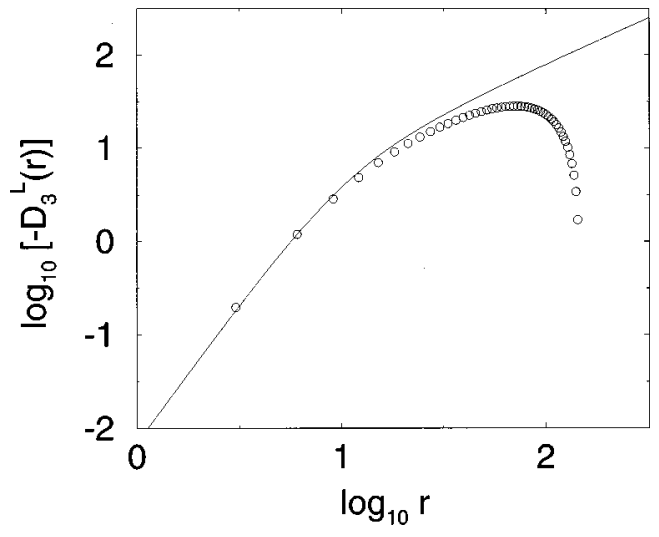

FIG. 2. Third order structure function from our numerical simulation (circles), compared with the one following from the Batchelor parametrization of $D_{2}^{L}(r)$ (full line). The Taylor-Reynolds number is $\operatorname{Re}_{\lambda}=110$.

adding a corresponding term to that equation as, e.g., done in Ref. [25].

\section{HOW TO APPLY ESS?}

We now plot the second vs the third order structure function in a compensated ESS type plot, i.e., $D_{2}^{L} /\left(D_{3}^{L}\right)^{2 / 3}$ vs $D_{3}^{L}$, see Fig. 3. The scaling regime in the numerical simulation is by far too short to identify any scaling exponent. However, if we repeat this plot, but now with $D_{3}^{* L}$ rather than $D_{3}^{L}$, ESS is seen, see Fig. 4. The reason is that $D_{2}^{L}$ and $D_{3}^{* L}$ have the same type of large scale saturation (i.e., becoming constant), whereas $D_{3}^{L}$ has a different type of large scale behavior (namely, dropping to zero). We have to conclude that the extension of the scaling regime by using ESS is mainly an extension towards large scales. This even holds if $\operatorname{Re}_{\lambda}$ is so small that there is no ISR yet. The small scale onset of scaling still is around $r \sim 10 \eta$, whether plotting $D_{2}$ vs $D_{3}^{* L}$ or vs

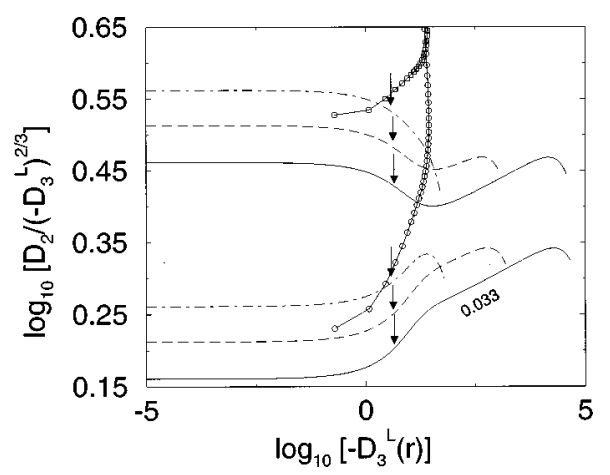

FIG. 3. Compensated ESS type plot $D_{2} /\left(D_{3}^{L}\right)^{2 / 3}$ vs $D_{3}^{L}$ for the structure functions from the numerical simulation (circles: longitudinal; squares: transversal) and those following from the Batchelor parametrization (3) of $D_{2}^{L}$ for Reynolds numbers $\mathrm{Re}=5.2 \times 10^{2}$ as in the numerical simulation, for $R e=5.2 \times 10^{4}$, and for $\operatorname{Re}=5.2 \times 10^{6}$, corresponding to Taylor-Reynolds numbers of $\operatorname{Re}_{\lambda}=90$ (dot-dashed), $\operatorname{Re}_{\lambda}=900$ (dashed), and $\operatorname{Re}_{\lambda}=9000$ (solid); $\zeta=0.7, b^{L}=2.0$. The three lower curves are for the longitudinal structure functions, the three upper ones for the transversal ones. The arrows indicate $10 \eta$. The external length scales $L$ are beyond the regimes shown.

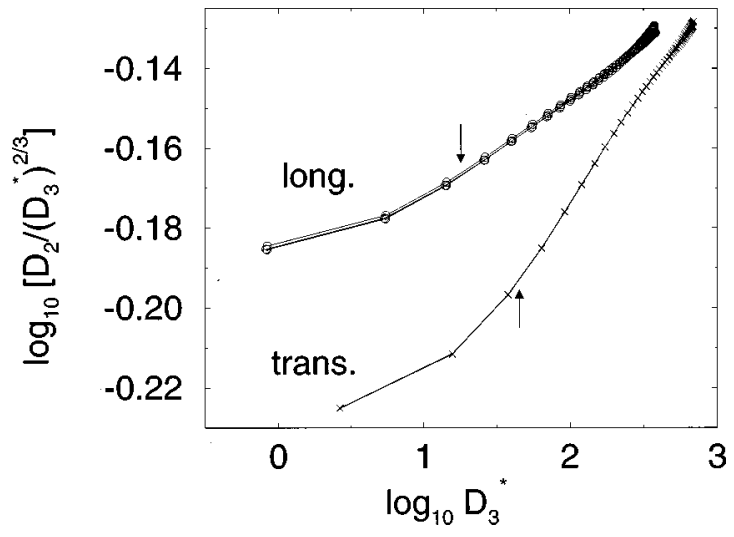

FIG. 4. Compensated ESS type plots $D_{2}^{L} /\left(D_{3}^{* L}\right)^{2 / 3}$ vs $D_{3}^{* L}$ (three different data sets for three different space directions which, however, agree very well) and $D_{2}^{T} /\left(D_{3}^{* T}\right)^{2 / 3}$ vs $D_{3}^{* T}$ (two data sets, also agreeing) for the numerical turbulence, $\operatorname{Re}_{\lambda}=110$. The arrows again indicate $10 \eta$; the data points are for $r=3 \eta, r=6 \eta, r=9 \eta, \ldots$, left to right.

$r$, which is roughly the crossover scale $a \approx 12.4 \eta$ found from employing Eq. (3). In their numerical simulation Briscolini et al. [3] find an ESS extension down to $r \sim 7 \eta$ (see Fig. 4 of their paper), roughly the same as the $10 \eta$ reported here, but slightly smaller. As pointed out to us by Benzi, the origin of the slight difference may be that the small scale resolution in Briscolini et al. [3] is down to $1 \eta$, whereas here we only have a $3 \eta$ resolution and the deviations in the structure functions due to the lower end of the resolution may influence the scaling exponents in a certain range of larger scales.

Our next point is to advocate compensated ESS plots for the visualization of intermittency effects. Already Meneveau [16]—see Fig. 1 of that paper-reveals how misleading an ESS plot $D_{p}^{L}$ vs $D_{3}^{* L}$ can be. Here, we demonstrate this in Fig. 5(a), which shows the original ESS plot $D_{2}$ vs $D_{3}^{*}$, only
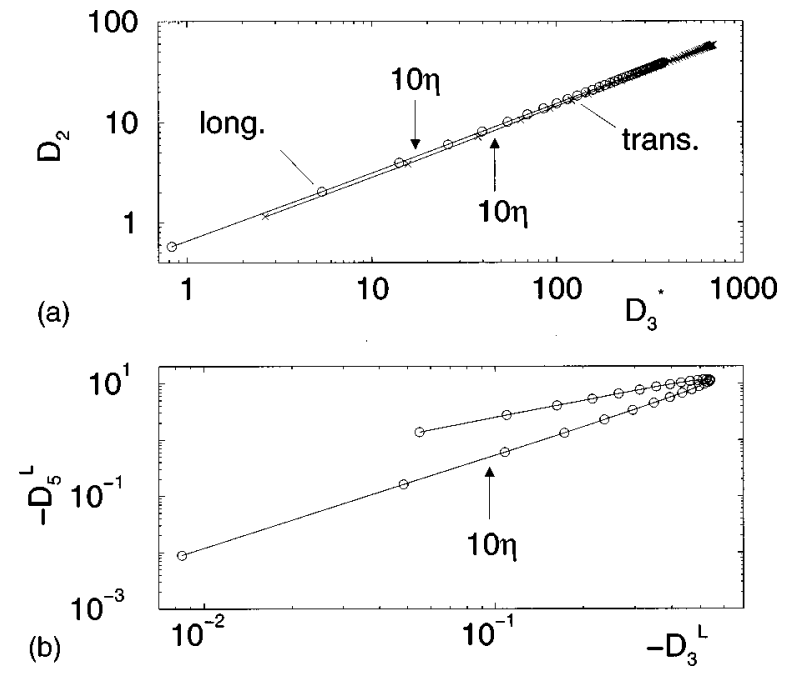

FIG. 5. (a) ESS plots $D_{2}(r)$ vs $D_{3}^{*}(r)$ for the longitudinal and transversal structure functions; $\operatorname{Re}_{\lambda}=110$. The data are the same as in the previous figure, where we showed the compensated ESS type plots, in which the different behavior in the VSR and the ISR is clearly visible. (b) ESS plots for $-D_{5}^{L}(r)$ vs $-D_{3}^{L}(r)$. In this figure the Taylor-Reynolds number is only 70 , but we checked that the lack of ESS does not decrease with increasing $\operatorname{Re}_{\lambda}$. 

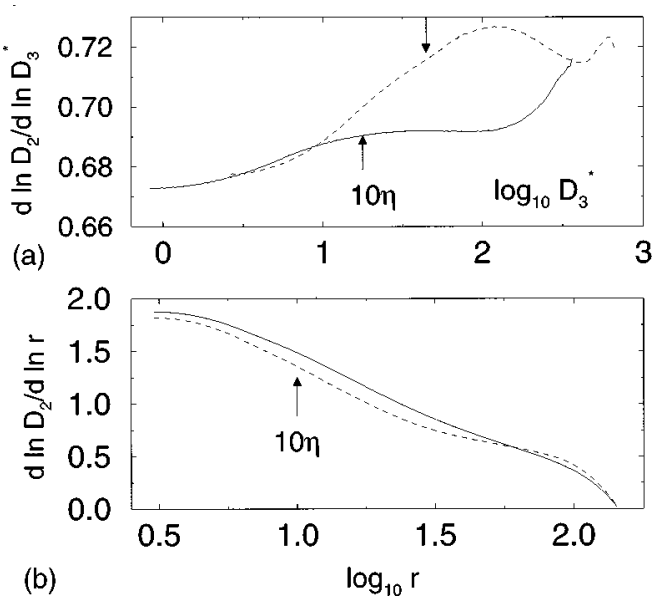

FIG. 6. (a) Local slopes $d \log _{10} D_{2}^{L} / d \log _{10} D_{3}^{* L}$ (solid) and $d \log _{10} D_{2}^{T} / d \log _{10} D_{3}^{* T}$ (dashed) of the curves in Fig. 5(a). The arrows indicate $10 \eta$. (b) Local slopes $d \log _{10} D_{2}^{L} / d \log _{10} r$ (solid) and $d \log _{10} D_{2}^{T} / d \log _{10} r$ (dashed) of the numerical data curves $D_{2}^{L, T}(r)$ in Fig. 1.

pretending better scaling than in Fig. 4. The reason is that in the VSR the scaling exponent is $2 / 3$ for trivial reasons, a value which can hardly be distinguished by eye from the ISR value $2 / 3+\delta \zeta_{2} \approx 0.70$. To avoid this similarity of the VSR and ISR exponents, we prefer to use compensated ESS plots $[26,11]$.

If one plots local slopes [8] as done in Fig. 6(a) it of course does not make any difference whether one takes them from compensated ESS plots or standard ESS plots. From Fig. 6(a) one notices that the ESS scaling $\xi_{2}^{L} \approx 0.69$ and $\xi_{2}^{T} \approx 0.72$ begins around $10 \eta$. From Fig. 6(b) one also notices that without ESS one could not deduce any scaling exponent at all for the small Reynolds number of our numerical calculation. ESS is thus useful already for the simple reason that a transition from a local slope of $2 / 3$ to roughly 0.70 is shorter than from a local slope 2 to roughly 0.70 .

As we will show now, there is no extended scaling regime towards scales much smaller than order $\sim 10 \eta$, either, if one does ESS type plots with $D_{3}^{L}$ instead of $D_{3}^{* L}$. We do so by plotting $D_{2}^{L} /\left(D_{3}^{L}\right)^{2 / 3}$ vs $D_{3}^{L}$ with $D_{3}^{L}$ following [via Eq. (2)] from the Batchelor parametrization Eq. (3) of $D_{2}^{L}$ for various Re, see Fig. 3. We observe three regimes: The VSR without any scaling corrections (i.e., a horizontal line in Fig. 3), a crossover regime, corresponding to the range from $r \sim 1 \eta$ to $r \sim 10 \eta$, and only for large scales and large $\operatorname{Re} \gtrsim 500$ the ISR scaling corrections $\delta \zeta_{2}=0.033$ can be identified.

To summarize this subsection: There seems to be ESS towards large scales, if the structure functions plotted against each other are both calculated with the moduli, i.e., have the same large scale saturation behavior. In particular, for the third order longitudinal structure function this means that it is essential to take $D_{3}^{* L}$ rather than $D_{3}^{L}$ and to clearly distinguish between the $\zeta_{p}$ and $\xi_{p}$ exponents. This was already stressed by Stolovitzky and Sreenivasan [8], see in particular their figures 2 and 4 where they compare local slopes of $D_{8}^{L}$ vs $D_{3}^{L}$ and $D_{3}^{* L}$.

The natural question to ask is, is there also ESS for odd order structure functions (calculated without the modulus) plotted against each other? In Fig. 5(b) we plot $-D_{5}^{L}$ vs $-D_{3}^{L}$. No ESS towards large scales is seen. It seems that odd and even order moments obey fundamentally different types of statistics. This finding may be connected to Herweijer and van de Water's finding [27] that $\zeta_{p}$ for odd $p$ [calculated from $D_{p}^{L}(r) \propto r^{\zeta}$ ] are smaller than expected from an extrapolation of the neighboring $\zeta_{p \pm 1}$ (for which the $p \pm 1$ are even). A difference between the $\zeta_{p}$ for odd and even $p$ was also found by Stolovitzky et al.[14,8]; however, for the flow analyzed in those references the $\zeta_{p}$ for odd $p$ are larger than expected from the extrapolation.

We do not understand why nonuniversal forcing and large scale boundary effects roughly cancel out in even order structure functions but not in odd order ones (calculated without the modulus). With a more elaborate technique it may even be possible to extract nonuniversal properties also from ESS plots of even structure functions. On the other hand, we cannot exclude that there is more universality in
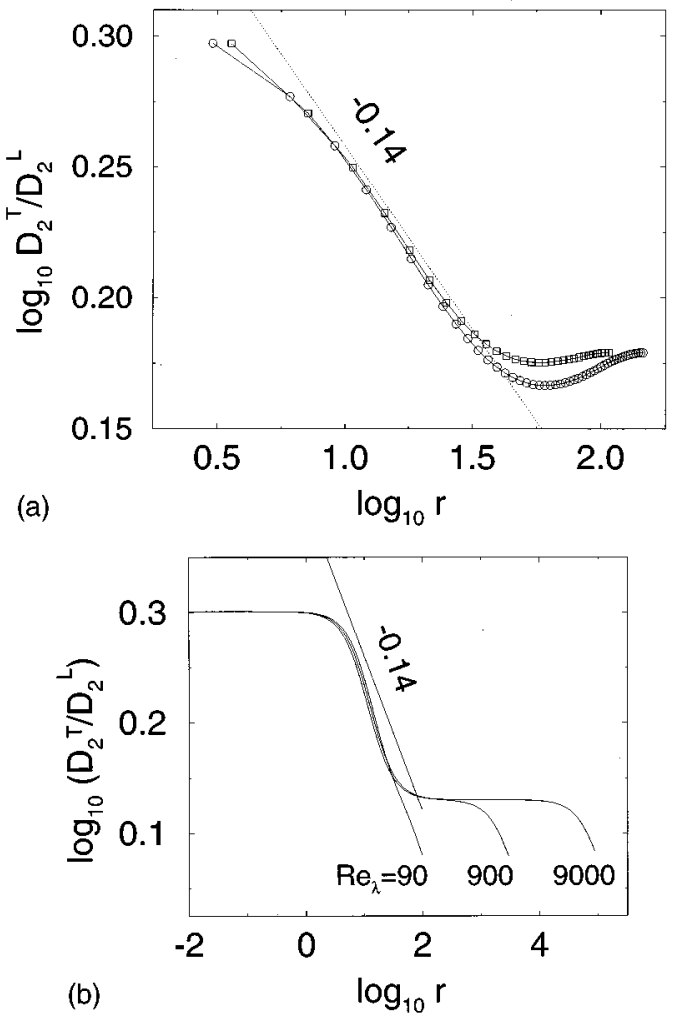

FIG. 7. (a) The ratio $D_{2}^{T}(r) / D_{2}^{L}(r)$ vs $r$ for the numerical data of a $\operatorname{Re}_{\lambda}=110$ (circles) and a $\operatorname{Re}_{\lambda}=70$ (squares) simulation. Erroneously, one may deduce different scaling of $D_{2}^{L}(r)$ and $D_{2}^{T}(r)$ because the ratio depends on $r$. We also included an apparent slope of -0.14 . (b) $D_{2}^{T}(r) / D_{2}^{L}(r)$ as a function of $r$ for the Batchelor parametrization (3) for Taylor-Reynolds numbers of $\operatorname{Re}_{\lambda}=90$, $\operatorname{Re}_{\lambda}=900$, and $\operatorname{Re}_{\lambda}=9000$. The plateau in the ISR (at roughly $\log _{10}(4 / 3)=0.125$ ) expected for isotropic homogeneous flow only starts to develop for as large $\operatorname{Re}_{\lambda}$ as $\operatorname{Re}_{\lambda} \sim 500$. The VSR plateau is at $\log _{10} 2=0.30$. The $r$-dependent intermediate range characterizes the transition between the VSR and the ISR. The slope is related to its width and the different heights of the two plateaus. As a side remark we mention that in this plot one can also notice the $\operatorname{Re}_{\lambda}$ dependence of the VSR-ISR crossover $a$, cf. Eq. (7): the transition range is shifted towards smaller $r$ with increasing $\operatorname{Re}_{\lambda}$. 
decaying turbulence where less anisotropy through the forcing and the boundaries is felt.

\section{LONGITUDINAL VS TRANSVERSAL STRUCTURE FUNCTIONS}

Next, we focus on the difference in the scaling between longitudinal and transversal structure functions, $D_{p}^{L}(r) \propto r^{\zeta_{p}^{L}}$ and $D_{p}^{T}(r) \propto r_{p}^{\zeta_{p}^{T}}$, respectively. Recently, different degrees of intermittency for longitudinal and transversal fluctuations were reported in some experiments $[24,28,29]$ and numerical simulations on decaying turbulence [30]. We confirmed these findings for statistically stationary turbulence [11] (see also Ref. [31]). More precisely, it was the ESS type scaling exponents $\xi_{p}^{L}$ and $\xi_{p}^{T}$, defined by $D_{p}^{L} \propto\left(D_{3}^{* L}\right)^{L}$ and $D_{p}^{T}$ $\propto\left(D_{3}^{* T}\right)^{\xi_{p}^{T}}$, which are clearly different; we found $\delta \xi_{6}^{L}=0.21 \pm 0.01$ and $\delta \xi_{6}^{T}=0.43 \pm 0.01$ for the deviations from the mean field value $\xi_{6}=2[11]$.

One would be tempted to conclude that the best way to see a deviation in scaling between $D_{p}^{L}$ and $D_{p}^{T}$ would be to plot the ratio $D_{p}^{T} / D_{p}^{L}$ vs $r$ (or vs $D_{3}^{L}$ ). According to Eq. (6), $D_{2}^{T}(r)$ and $D_{2}^{L}(r)$ scale the same in the ISR, i.e., the ratio should be constant. However, Fig. 7(a) seems to imply different scaling of $D_{2}^{L}(r)$ and $D_{2}^{T}(r)$.

The reason for this apparent discrepancy is that the argument of equal scaling of $D_{2}^{L}(r)$ and $D_{2}^{T}(r)$ is only valid if both structure functions scale individually. This is not the case in the transition ranges or if there is not any ISR yet. Here, the Reynolds number achieved in the full simulation is by far too small to give the asymptotic (ISR) scaling. In Fig. 7(b) we redo this type of plot, but now within the Batchelor parametrization for which we can achieve arbitrarily large $\operatorname{Re}_{\lambda}$. Only if $\operatorname{Re}_{\lambda} \geq 500$ a plateau starts to develop, showing the onset of the asymptotically correct ISR behavior. To reliably determine scaling exponents from the plateau, one would need at least Taylor-Reynolds numbers $\sim 1000$ and beyond. For $\operatorname{Re}_{\lambda} \sim 100$ there is a fake scaling law with an apparent exponent of -0.14 , which has nothing to do with inertial range scaling.

Going back to Eqs. (3) and (6), this behavior can be un- derstood. In the VSR we must have $D_{2}^{T} / D_{2}^{L}=2$ [because of Eq. (6) and $\left.D_{2}^{L} \propto r^{2}\right]$ and in the ISR we have $D_{2}^{T} / D_{2}^{L} \approx 4 / 3$ [because of Eq. (6) and roughly $D_{2}^{L} \propto r^{2 / 3}$ ], just as seen in Fig. 7(b). The crossover between these two regimes is about a decade. The same can be seen from Fig. 3 where besides $D_{2}^{L} /\left(D_{3}^{L}\right)^{2 / 3}$ we also plotted $D_{2}^{T} /\left(D_{3}^{L}\right)^{2 / 3}$ vs $D_{3}^{L}$. In the crossover regime where the former curve bends up, the latter bends down. Again, only for $\operatorname{Re}_{\lambda} \gtrsim 500$ does the asymptotic scaling exponents $\delta \zeta_{2}^{L}=\delta \zeta_{2}^{T}=0.033$ start to be observable.

The same finite $\operatorname{Re}_{\lambda}$ effects which we discussed for the second order structure functions, where $D_{2}^{L}$ and $D_{2}^{T}$ are known to have the same scaling, will hinder determination of scaling exponents vs $r$ (or vs $D_{3}^{L}$ ) in higher order structure functions for too low $\operatorname{Re}_{\lambda} \lesssim 500$.

\section{SUMMARY}

To conclude, we confirmed the finding of Briscolini et al. [3] that ESS does not extend to scales below order $\sim 10 \eta$. We furthermore showed from calculations with the Batchelor parametrization that scaling exponents $\zeta_{p}$ calculated from structure functions plotted vs $r$ (or vs $D_{3}^{L}$ ) can only securely be measured for $\operatorname{Re}_{\lambda}$ sufficiently larger than 500. For smaller $\mathrm{Re}_{\lambda}$, in particular for all present-day numerical simulations, one is restricted to relative, ESS type scaling exponents $\xi_{p}$ calculated from ESS type plots $D_{p}^{* L}(r)$ vs $D_{q}^{* L}(r)$ and $D_{p}^{* T}(r)$ vs $D_{q}^{* T}(r)$, whereby it is essential to calculate the structure functions from the moduli of the velocity differences. For odd order moments, calculated without taking the modulus, ESS does not hold in the presented numerical simulation.

\section{ACKNOWLEDGMENTS}

We thank Roberto Benzi and Luca Biferale for very helpful comments on the manuscript. Support for this work by the Deutsche Forschungsgemeinschaft (DFG) under Grant No. D3 of SBF185 and by the German-Israeli Foundation (GIF) is gratefully acknowledged. The HLRZ Jülich supplied us with computer time.
[1] R. Benzi et al., Phys. Rev. E 48, R29 (1993).

[2] R. Benzi, S. Ciliberto, C. Baudet, and G. R. Chavarria, Physica D 80, 385 (1995).

[3] M. Briscolini, P. Santangelo, S. Succi, and R. Benzi, Phys. Rev. E 50, R1745 (1994)

[4] A. S. Monin and A. M. Yaglom, Statistical Fluid Mechanics (The MIT Press, Cambridge, MA, 1975).

[5] M. Nelkin, Adv. Phys. 43, 143 (1994).

[6] U. Frisch, Turbulence (Cambridge University Press, Cambridge, England, 1995).

[7] Roberto Benzi (private communication) communicated to us that for an experimental flow in a regime where no forcing is felt this indeed was the case. Other authors find differences; e.g., J. A. Herweijer (Ph.D. thesis, University of Eindhoven,
$1995)$ finds $D_{3}^{L}(r) \propto r^{1.035 \pm 0.005}$ and $D_{3}^{* L}(r) \propto r^{1.055 \pm 0.005}$ for a wind tunnel measurement.

[8] G. Stolovitzky and K. R. Sreenivasan, Phys. Rev. E 48, R33 (1993).

[9] A. Arneodo et al., Europhys. Lett. 34, 411 (1996).

[10] F. Belin, P. Tabeling, and H. Willaime, Physica D 93, 52 (1996).

[11] S. Grossmann, D. Lohse, and A. Reeh, Phys. Fluids (to be published).

[12] G. K. Batchelor, Proc. Cambridge Philos. Soc. 47, 359 (1951).

[13] H. Effinger and S. Grossmann, Z. Phys. B 66, 289 (1987).

[14] G. Stolovitzky, K. R. Sreenivasan, and A. Juneja, Phys. Rev. E 48, R3217 (1993); L. Sirovich, L. Smith, and V. Yakhot, Phys. Rev. Lett. 72, 344 (1994). 
[15] D. Lohse and A. Müller-Groeling, Phys. Rev. Lett. 74, 1747 (1995).

[16] Ch. Meneveau, Phys. Rev. E 54, 3657 (1996).

[17] D. Lohse and A. Müller-Groeling, Phys. Rev. E 54, 395 (1996).

[18] R. Benzi et al., Physica D 96, 162 (1996).

[19] K. R. Sreenivasan, Phys. Fluids 7, 2778 (1995).

[20] K. R. Sreenivasan, Phys. Fluids 27, 1048 (1984).

[21] D. Lohse, Phys. Rev. Lett. 73, 3223 (1994).

[22] S. Grossmann, Phys. Rev. E 51, 6275 (1995); G. Stolovitzky and K. R. Sreenivasan, ibid. 52, 3242 (1995).

[23] A. Praskovsky and S. Oncley, Phys. Fluids 6, 2886 (1994).

[24] J. Herweijer and W. van de Water, in Advance in Turbulence
$V$, edited by R. Benzi (Kluwer Academic Publishers, New York, 1995), p. 210.

[25] V. Yakhot, Phys. Rev. Lett. 69, 769 (1992).

[26] S. Grossmann, D. Lohse, and A. Reeh, Phys. Rev. Lett. 77, 5369 (1996).

[27] J. Herweijer and W. van de Water, Phys. Rev. Lett. 74, 4651 (1995).

[28] W. van de Water and J. Herweijer (unpublished); Phys. Scr. T67, 136 (1996).

[29] R. Camussi and R. Benzi, Phys. Fluids 9, 257 (1997).

[30] O. N. Boratav and R. B. Pelz, Phys. Fluids 9, 1400 (1997).

[31] S. Chen, K. R. Sreenivasan, M. Nelkin, and N. Cao, Phys. Rev. Lett. 79, 2253 (1997). 